\title{
Cutting out the liver fat
}

\author{
Better animal models of nonalcoholic steatohepatitis are needed to more fully understand the disease and to identify \\ potential new therapeutic treatments for this increasingly common condition.
}

t's t's stated so often but is nonetheless true: there is a worldwide epidemic of obesity. As of 2016, an estimated 39\% of adults around the world were overweight, and $13 \%$ of adults, representing approximately 650 million individuals, were classified as obese. Although we and others have debated the issue of whether obesity itself should be classified as a pathology (see Nat. Med. 19, 947, 2013), what is clear is that obesity can increase the risk for multiple comorbidities, including many metabolic complications that can affect almost every major organ of the body. One of these critical organs is the liver, where a toxic buildup of fats in hepatocytes due to obesity can lead to progression from simple hepatic steatosis (also known as 'fatty liver disease', which is considered a benign condition) to nonalcoholic steatohepatitis (NASH). The latter condition is characterized by inflammation of the liver and, in its later stages, fibrotic scar tissue; it can progress further to cirrhosis and to liver cancer, ultimately leading to end-stage organ failure. The umbrella term for this panoply of conditions, from simple steatosis through cirrhosis, when it occurs in the absence of excessive alcohol consumption is nonalcoholic fatty liver disease (NAFLD), and it has become a major concern in the clinic. In the US, it is estimated that $25 \%$ of the overall population, roughly 75 million people, have some form of NAFLD and that roughly $6-30 \%$ of individuals with simple steatosis progress to NASH. And with the increasing incidence of obesity, especially if type 2 diabetes also is present, the occurrence of NAFLD is likely to increase markedly. Currently, there is a lack of effective pharmacological treatments for the different aspects of NAFLD, including NASH.

There are a number of factors that have hindered breakthroughs in treatment of the different stages of NAFLD. These include the lack of relatively accurate animal models, the need for invasive tests (notably, liver biopsies) to properly diagnose the condition in patients and an unclear understanding of the progression of NAFLD in humans. For example, the course of pathology as described above is not always stepwise, as some patients appear to skip over the stage of cirrhosis and go straight from NASH to liver cancer while others progress to liver cancer without developing notable fibrosis. Also, it is not clear why many individuals with 'benign' fatty liver disease never develop NASH.

The etiology of NASH is still quite murky. NASH itself was not described in the clinic until 1980, and in the early days the accepted rodent model was use of a methionine- and choline-deficient diet, as this was found to induce liver fibrosis. But rodents actually lose weight on this diet and do not display hepatic insulin resistance, which is known to promote excessive fat accumulation in the liver. Thus, this animal model is not reflective of most cases of obesity-associated NASH in humans. A more recently accepted mouse model combines a high-fat diet with a high-cholesterol diet. This regimen leads to what appears to be NASH; still, the progression in mice is slow, and the degree of liver fibrosis is not as severe as that seen in the later stages of NASH in humans. Others have tried creating a better mouse model of NASH by also incorporating high levels of fructose in normal chow; using a limited amount of a fibrosis-inducing agent such as bleomycin along with a high-fat diet; or raising the mice at thermoneutrality to reduce their stress and thus allow for a more potent immune response, a key contributor to the pathology in NASH. A combination of these approaches may ultimately lead to a more relevant rodent model of NASH. But what will be needed to prove the accuracy of the model is not simply pathology to identify the degree of immune infiltration and fibrosis in the liver, but also use of genetic analyses on the hepatocytes to see whether the resulting gene expression signature of the model accurately reflects that seen in human hepatocytes from individuals with NASH.

The current rodent models of NASH, particularly those in mice owing to the ability to facilitate genetic manipulations in this species, have allowed us to gain important insight into NASH progression, including identifying a role for several stress-related kinases-ASK1, p38-MAPK and JNK-in promoting inflammation. Even so, what would likely allow for more assured translation to clinical trials would be a large animal model that is more closely related to humans-notably, nonhuman primates. Such models have recently been reported and involve the selection of monkeys with spontaneously occurring metabolic syndrome (a constellation of pathologies including obesity, hyperglycemia, dyslipidemia and elevated blood pressure) and mild NASH that are then placed on a high-fat diet to induce more severe NASH. It is still unclear whether such a nonhuman primate model can be easily generated or reproduced, but the availability of primate models may more rapidly advance the potential therapeutic options in development. These potential therapies have been limited to anti-inflammatory and antioxidant approaches, such as pioglitazone or vitamin E (the PIVENS trial), a synthetic bile acid (obeticholic acid in the REGENERATE trial) and a dual peroxisome proliferator-activated receptor $\alpha / \delta$ agonist (elafibranor in the RESOLVE-IT trial).

More clinical trials for the treatment of NASH are needed, but it should be noted that such trials still have limitations. Currently, patients with NASH need to be identified by liver biopsy, which limits ease of enrollment and therefore drives up cost and the amount of time invested, and continued biopsies are needed to monitor drug response. These hurdles are all the more reason to strive for more accurate animal models that can be used to test the potential effectiveness of a therapy for NASH. Doing so should make the leap to a clinical trial more productive. 